\title{
V-shaped dark solitons in inhomogeneous optical fibers
}

\author{
Guoli Ma*, Xin Zhang, Xunli Zhang, and Hongyan Guo \\ Institute of Aeronautical Engineering, Binzhou University, \\ Binzhou 256603, China
}

\begin{abstract}
Soliton control offers novel conceptual opportunities for all-optical shaping, switching and routing of optical signals encoded in soliton formats. In this paper, V-shaped dark solitons propagation in inhomogeneous optical fibers are investigated to carry out soliton control. Analytic dark soliton solutions for the variable coefficient nonlinear Schrödinger equation are obtained with the Hirota method. The group velocity dispersion coefficient of inhomogeneous optical fibers is chosen to be the tanh function, and the influences of corresponding parameters are analyzed. Results might be of potential applications in the design of all optical switches and pulse signal converters in ultra-fast optics.
\end{abstract}

Keywords: Solitons, dark solitons, all optical switches, variable coefficient nonlinear Schrödinger equation

${ }^{*}$ Corresponding author, with e-mail address as bz-mgl@163.com 


\section{Introduction}

Since the theoretical prediction and experimental confirmation of optical solitons $[1,2]$, they have been the research object of ultra-fast optics, optical communication and all-optical ultrafast switching devices [3-12]. Among those applications, soliton control is one of the important issues to investigate universal phenomena and provide a unique exploration tool, and some attention has been paid to study soliton control [13-22].

On the one hand, soliton control for bright solitons has been extensively studied [13-22]. Some types of solitons have been obtained with the different choices of the group-velocity dispersion (GVD) of inhomogeneous optical fibers [13-15]. Interactions between butterflyshaped pulses have been investigated to improve pulse qualities in inhomogeneous optical fibers [16]. Soliton control and management using generalized external potentials in inhomogeneous optical fibers have been presented based on solving the variable-coefficient nonlinear Schrödinger (vcNLS) equation [17]. Besides, the manipulation of the deceleration or acceleration of soliton emitted from Airy pulse has been studied [18]. Controlling temporal solitons in the generalized inhomogeneous coupled NLS equations with varying source terms have been observed [19], and controllable combined Peregrine soliton and Kuznetsov-Ma soliton in PT-symmetric nonlinear couplers with gain and loss have been obtained [20]. The possibility of controlling the propagation of an intense optical pulse by means of a less powerful pulse has been demonstrated [21]. Moreover, soliton control in inhomogeneous nonlinear media with the parity-time symmetric potentials has been investigated numerically [22].

On the other hand, the study of dark solitons has been attractive and active, and great progress has been made [23-28]. Dark solitons have been observed in the normal dispersion regime [23], and soliton interaction and soliton control between bright and dark solitons have been presented [24]. Dark soliton formation in erbium-doped fiber lasers has been experimentally demonstrated [25], and the experimental observation of soliton-dark pulse pair formation in a birefringent cavity fiber laser has been reported [26]. The formation of a pair of dark solitons from a single nonlinear black spin-wave pulse have been observed [27]. Furthermore, dark solitons in dual-core waveguides with dispersive coupling have been obtained [28].

However, the V-shaped dark solitons propagation in inhomogeneous optical fibers has not been reported when the GVD coefficient of inhomogeneous optical fibers is the tanh function. In this paper, we will study the V-shaped dark solitons propagation in inhomogeneous optical fibers, which can used to carry out soliton control. The dark soliton solutions will be obtained. By selecting the corresponding parameters of inhomogeneous optical fibers, influences of them will be presented.

This paper will be structured as follows. In Section II, analytic dark soliton solutions will be derived. In Section III, influences of corresponding parameters will be analyzed. Finally, our conclusions will be made in Section IV. 


\section{Analytic dark soliton solutions}

The propagation of optical solitons in inhomogeneous optical fibers can be described by the vcNLS equation [29-31]:

$$
\frac{\partial u(z, t)}{\partial z}-i \frac{D(z)}{2} \frac{\partial^{2} u(z, t)}{\partial t^{2}}+i \rho(z)|u(z, t)|^{2} u(z, t)=i g(z) u(z, t),
$$

where $u(z, t)$ is the temporal envelope of solitons. $z$ is the longitudinal coordinate, and $t$ is the time in the moving coordinate system. $D(z)$ represents the GVD coefficient, $\rho(z)$ is the coefficient of nonlinearity, and $g(z)$ is related to the losses or gain coefficient.

To construct the soliton solutions, we perform the dependent variable transformation [32]

$$
u(z, t)=\frac{h(z, t)}{f(z, t)}
$$

where $h(z, t)$ is a complex differentiable function, and $f(z, t)$ is a real one. With the transformation, the resulting bilinear forms for Eq. (1) can be derived as

$$
\begin{aligned}
& D_{z} h \cdot f-\frac{i}{2} D(z) D_{t}^{2} h \cdot f-i[g(z)-\lambda(z)] h \cdot f=0, \\
& D(z) D_{t}^{2} f \cdot f-2 \lambda(z) f f+2 \rho(z) h h^{*}=0 .
\end{aligned}
$$

$D_{z}$ and $D_{t}[33]$ are the Hirota's bilinear operators, which can be defined by

$$
D_{z}^{m} D_{t}^{n}(H \cdot F)=\left.\left(\frac{\partial}{\partial z}-\frac{\partial}{\partial z^{\prime}}\right)^{m}\left(\frac{\partial}{\partial t}-\frac{\partial}{\partial t^{\prime}}\right)^{n} H(z, t) F\left(z^{\prime}, t^{\prime}\right)\right|_{z^{\prime}=z, t^{\prime}=t}
$$

where $m$ and $n$ are the positive integers, $H$ is the function of $z$ and $t$, and $F$ is the function of the formal variables $z^{\prime}$ and $t^{\prime}$.

To solve bilinear forms $(3)-(4), h(z, t)$ and $f(z, t)$ can be expanded with respect to the following power series expansions:

$$
\begin{aligned}
& h(z, t)=h_{0}(z, t)\left[1+\varepsilon h_{1}(z, t)\right], \\
& f(z, t)=1+\varepsilon f_{1}(z, t),
\end{aligned}
$$

where $\varepsilon$ is a formal expansion parameter, $h_{0}(z, t), h_{1}(z, t)$ and $f_{1}(z, t)$ are the differentiable functions. Substituting expressions (6)-(7) into bilinear forms (3)-(4) and equating coefficients of the same powers of $\varepsilon$ to zero yield the recursion relations for $h_{0}(z, t), h_{1}(z, t)$ and $f_{1}(z, t)$. Then, analytic soliton solutions for Eq. (1) can be obtained.

To obtain soliton solutions, we assume that

$$
\begin{aligned}
& h_{0}(z, t)=\exp \left[i m_{1}(z) z+i n_{1} t\right], \\
& h_{1}(z, t)=-\exp \left\{\left[a_{11}(z)+i a_{12}(z)\right] z+\left(b_{11}+i b_{12}\right) t+\left(k_{11}+i k_{12}\right)\right\}, \\
& f_{1}(z, t)=\beta \exp \left\{\left[a_{11}(z)+i a_{12}(z)\right] z+\left(b_{11}+i b_{12}\right) t+\left(k_{11}+i k_{12}\right)\right\},
\end{aligned}
$$


where $m_{1}(z), a_{11}(z)$ and $a_{12}(z)$ are differentiable functions to be determined, $n_{1}, b_{11}, b_{12}, k_{11}$, $\beta$ and $k_{12}$ are the real constants. Substituting $h_{0}(z, t)$ and $h_{1}(z, t)$ into bilinear form (3), and collecting the coefficient of $\varepsilon$, we get the constraints on the parameters:

$$
\begin{aligned}
& m_{1}(z)=\frac{\int\left[2 g(z)-n_{1}^{2} D(z)-2 \rho(z)\right] d z}{2 z}, a_{11}(z)=-b_{11} n_{1} \frac{\int D(z) d z}{z}, \\
& b_{12}=0, k_{12}=0, a_{12}(z)=0, \rho(z)=\beta b_{11}^{2} D(z) / 2(1+\beta)
\end{aligned}
$$

Without loss of generality, we set $\varepsilon=1$, and analytic dark soliton solutions can be expressed as

$$
u(z, t)=\frac{h(z, t)}{f(z, t)}=\exp \left\{\int\left[i g(z)-\frac{i}{4}\left(b_{11}^{2}+2 n_{1}^{2}\right) D(z)\right] d z+i n_{1} t\right\} \frac{e^{b_{11} n_{1} \int D(z) d z}-e^{b_{11} t+k_{11}}}{e^{b_{11} n_{1} \int D(z) d z}+e^{b_{11} t+k_{11}}} .
$$

\section{Discussion}

According to expression (9), we choose the parameters as $n_{1}=-5.49, b_{11}=-4.3$, $k_{11}=8.95, h(z)=1$, and $D(z)=-0.3 \operatorname{Tanh}(6.6 z)$, and can obtain the V-shaped dark solitons as shown in Figure 1 $(a)$. The propagation direction of solitons is suddenly changed at zero point, and maintain the original pulse duration and velocity. This may be applications in the design of all optical switches.
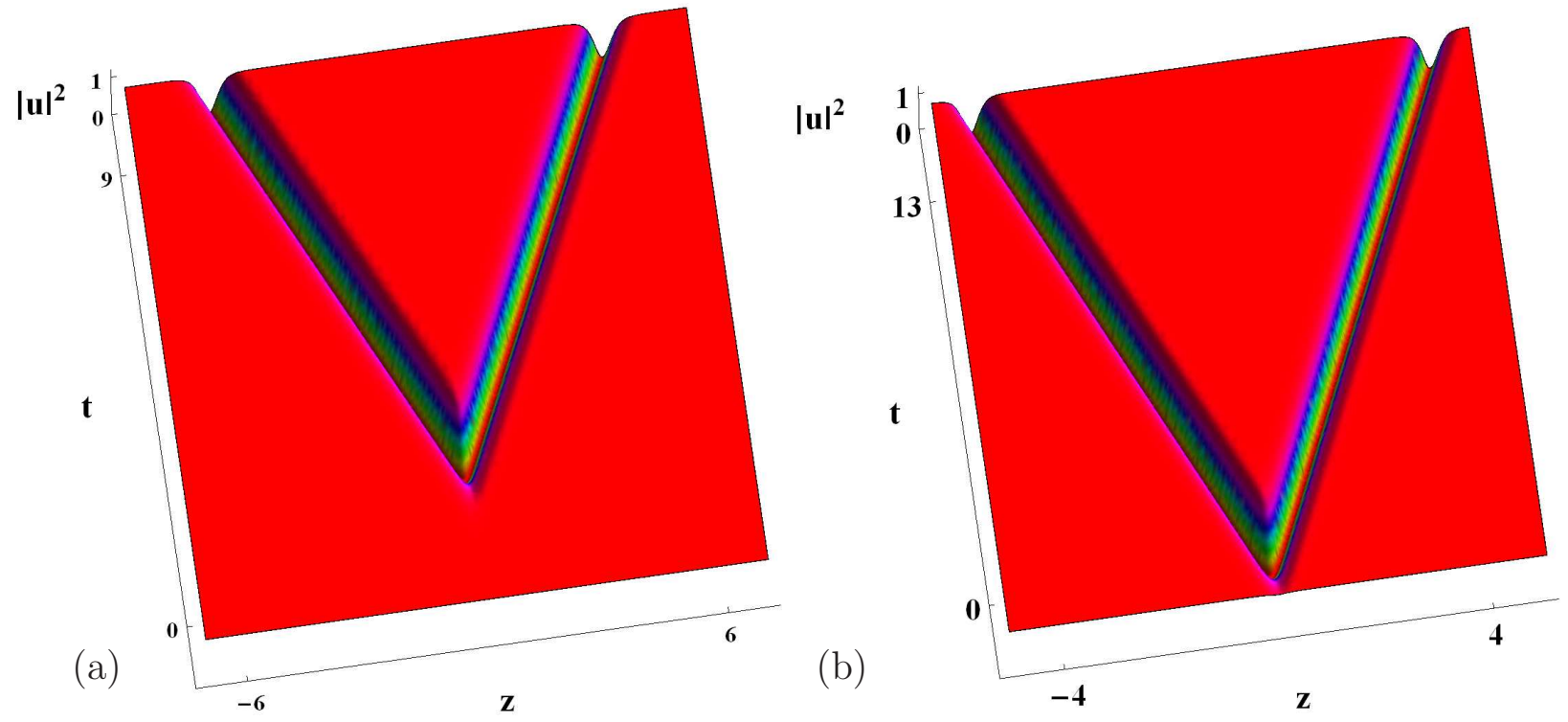

Figure 1. V-shaped dark solitons propagation in inhomogeneous optical fibers. Parameters are: (a) $n_{1}=-5.49, b_{11}=-4.3, k_{11}=8.95, h(z)=1$, and $D(z)=-0.3 \operatorname{Tanh}(6.6 z)$; (b) $n_{1}=-1.02$, $b_{11}=2.8, k_{11}=1.01, h(z)=1$, and $D(z)=-3.6 \operatorname{Tanh}(6.6 z)$.

The formation of V-shaped dark solitons is due to the sudden changes of GVD of inhomogeneous optical fibers. Adjusting the corresponding parameters of inhomogeneous optical fibers, we can change the pulse duration and velocity as shown in Figure 1(b). Besides, the propagation direction of solitons can be controlled in Figure 2. From expression (9), the 
pulse duration and velocity are related to $n_{1}, b_{11}$ and $D(z)$. Compared to Figures 1 and 2, the propagation direction of solitons is associated with the sign of $n_{1}$. In Figrue 1, the sign of $n_{1}$ is negative, while the sign of $n_{1}$ is positive in Figrue 2. Their propagation direction is opposite. Moreover, as the absolute value of the coefficient of tanh function increase, the soliton velocity will get faster.
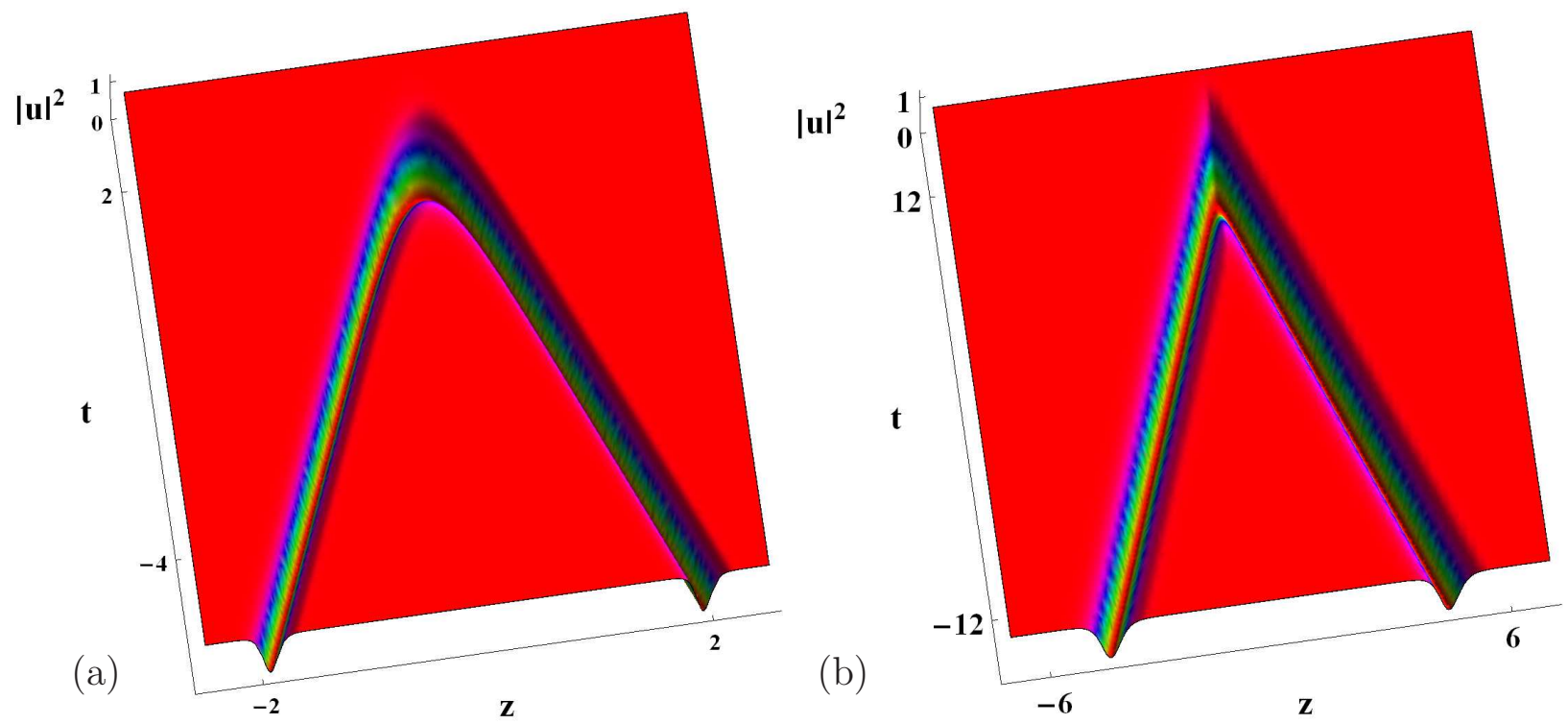

Figure 2. V-shaped dark solitons propagation in inhomogeneous optical fibers. Parameters are:

(a) $n_{1}=0.86, b_{11}=5.47, k_{11}=-6.6, h(z)=1$, and $D(z)=-5.0 \operatorname{Tanh}(2.8 z)$; (b) $n_{1}=6$, $b_{11}=1.08, k_{11}=-9.93, h(z)=1$, and $D(z)=-0.94 \operatorname{Tanh}(6.3 z)$.

\section{Conclusions}

V-shaped dark solitons propagation in inhomogeneous optical fibers have been investigated in this paper. With the help of the Hirota method, analytic dark soliton solutions (9) have been derived. When the GVD coefficient $D(z)$ has been chosen to be the tanh function, the $\mathrm{V}$-shaped dark solitons have been presented in Figures 1 and 2. The pulse duration and velocity have been related to the free parameters $n_{1}, b_{11}$, and $D(z)$. The propagation direction of solitons has been associated with the sign of $n_{1}$. Results in this paper can be used to the design of all optical switches in ultra-fast optics.

\section{Acknowledgements}

This work was supported by grants from Chinese National Science Foundation (Grant No. 41201368), Binzhou science and technology development plan (Grant No. 2013ZC0401) and Binzhou University scientific research fund project (Grant No. BZXYG1303) 


\section{References}

[1] Hasgawa A, Tappert F 1973 Appl. Phys. Lett. 23142

[2] Mollenauer L F, Stolen R H, Gordon J P 1980 Phys. Rev. Lett. 451095

[3] Guo R, Hao H Q 2014 Ann. Phys. 34410

[4] Guo R, Hao H Q, Zhang L L 2013 Nonlinear Dyn. 74701

[5] Guo R, Hao H Q 2013 Commun. Nonlinear Sci. Numer. Simulat. 182426

[6] Zhu H P, 2013 Nonlinear Dyn. 72873

[7] Wang Y Y, Dai C Q 2013 Nonlinear Dyn. 74429

[8] Tang B, Li D J, Tang Y 2014 Chaos 24023113

[9] Dai C Q, Zhu H P 2014 Ann. Phys. 341142

[10] Dai C Q, Zhu H P 2013 J. Opt. Soc. Am. B 303291

[11] Liu W J, Tian B, Lei M 2014 Appl. Math. Lett. 3028

[12] Dai C Q, Wang X G, Zhou G Q 2014 Phys. Rev. A 89013834

[13] Liu W J, Tian B, Zhang H Q 2008 Phys. Rev. E 78066613

[14] Liu W J, Tian B, Lei M 2013 Europhys. Lett. 10344002

[15] Liu W J, Han H N, Zhang L, Wang R, Wei Z Y, Lei M 2014 Laser Phys. Lett. 11045402

[16] Liu W J, Huang L G, Pan N, Lei M 2014 Ann. Phys. 349395

[17] Mahalingam A, Rajan M S M 2015 Opt. Fiber Technol. 2544

[18] Zhang L F, Liu K, Zhong H Z, Zhang J G, Deng J Q, Li Y, Fan D Y 2015 Sci. Rep. 5 11843

[19] Yang Y Q, Yan Z Y, Mihalache D 2015 J. Math. Phys. 56053508

[20] Dai C Q, Wang Y Y 2015 Nonlinear Dyn. 80715

[21] Tartara L 2015 J. Opt. Soc. Am. B 32395

[22] Hao R Y 2015 Opt. Commun. 338265

[23] Liu W J, Tian B, Zhang H Q, Xu T, Li H 2009 Phys. Rev. A 79063810 
[24] Liu W J, Tian B, Xu T, Sun K, Jiang Y 2010 Ann. Phys. 3251633

[25] Liu W J, Pang L H, Han H N, Tian W L, Chen H, Lei M, Yan P G, Wei Z Y 2015 Opt. Express 2326023

[26] Shao G D, Song Y F, Zhao L M, Shen D Y, Tang D Y 2015 Opt. Express 2326252

[27] Wang Z H, Cherkasskii M, Kalinikos B A, Wu M Z 2015 Phys. Rev. B 91174418

[28] Kartashov Y V, Konotop V V, Malomed B A 2015 Opt. Lett. 404126

[29] Agrawal G P 2007 Nonlinear Fiber Optics (San Diego, CA: Academic)

[30] Zheng H J, Wu C Q, Wang Z, Yu H S, Liu S L, Li X 2012 Optik 123818

[31] Zolotovskii I O, Novikov S G, Okhotnikov O G, Sementsov D I, Yavtushenko I O, Yavtushenko M S 2012 Opt. Spectrosc. 112893

[32] Liu W J, Tian B, Zhen H L, Jiang Y 2012 Europhys. Lett. 10064003

[33] Hirota R 1971 Phys. Rev. Lett. 271192 